\title{
An Active Contour Model for Segmentation Based on Cubic B-splines and Gradient Vector Flow
}

\author{
Matthias Gebhard, Julian Mattes, and Roland Eils \\ IBioS, German Cancer Research Center, D-69120 Heidelberg, Germany \\ m.gebhard@dkfz-heidelberg.de \\ http://www.dkfz-heidelberg.de/ibios
}

\begin{abstract}
The aim of this paper is to present advances in segmentation for visualization and quantitative analysis in bioimaging. Here, we combine two existing approaches for segmentation with snakes. Firstly, we use cubic B-splines to represent the snake using coarse-to-fine control point insertion; this allows to smooth adaptively the resulting contour while reducing the risk to get attracted from misdetected edges. Secondly, we put the snake in a gradient vector flow (GVF) field. This enables the snake to evolve into concavities of the shape. Further, sensitive parameters drop out in our setting and the attraction range with respect to initialization of the snake is enlarged.
\end{abstract}

\section{Introduction}

Imaging of cell biological objects in vivo leads often to a low signal to noise ratio as - because of their movement - a small capture time must be chosen in relation to the movement of objects [6]. For quantitative analysis of volume changes or surface deformation we need a robust and meaningful segmentation method which works semi-automatically by user interaction [4].

\section{Definitions and Notations}

To represent snake splines we use uniform B-splines where the basis functions are equidistantly located in the parameter space. A parameterized curve can be defined as $\mathbf{M}(u)=[x(u), y(u)], u \in[0, k]$, (here, $k \in \mathbb{N})$. The construction of a closed B-spline curve $\mathbf{M}(u)$ starts with the determination of $k$ control points $\mathbf{P}_{\mathbf{i}}, i=1, \ldots k, \mathbf{P}_{\mathbf{i}} \in \mathbb{R}^{2}$ (this also determines the range of the parameter interval).

We define $P=\left(\mathbf{P}_{\mathbf{i}}\right)^{T}$ built up from the control points $\mathbf{P}_{\mathbf{i}}, P \in \mathbb{R}^{k \times 2}$. For cubic B-splines the basis functions are of order three and differ only by translation [1]:

$$
\mathbf{M}(u)=\sum_{i=1}^{k} B(u, i) \mathbf{P}_{\mathbf{i}}
$$

One of the basic ideas of the primary snakes is the evolution of a contour over the image in search of minimizing a specific energy functional [2], traditionally defined by 

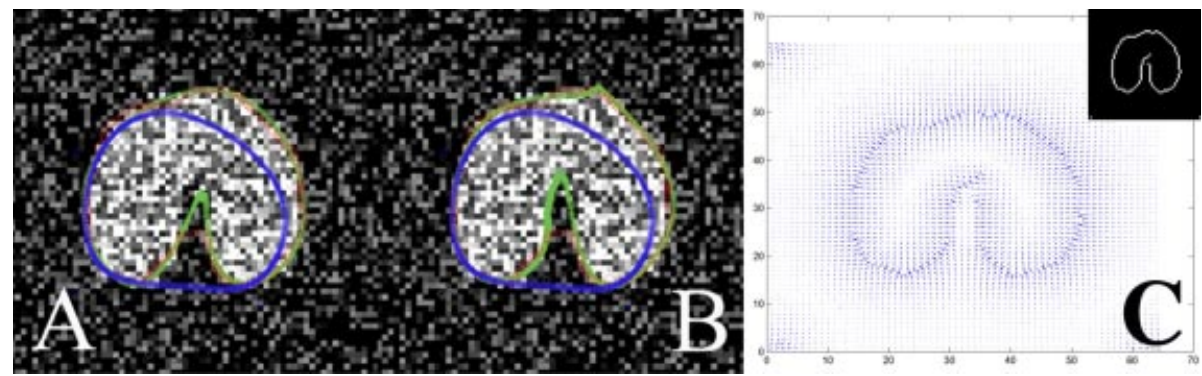

Fig. 1. Comparison between result obtained with the initial number of control points (CP) (A) and after CP insertion where the number of CP's are doubled (B). The blue line correspondes to the initial curve generated by the blue CP's, the red curve representing the polygon snake and the green shows the snake spline curve respective with its CP's (green). The GVF field corresponding to the Canny edge $(\mathrm{C})$ image

$$
E=\int_{0}^{k} \frac{1}{2}\left(\alpha\left|\mathbf{M}^{\prime}(u)\right|^{2}+\beta\left|\mathbf{M}^{\prime \prime}(u)\right|^{2}\right)+\gamma E_{\text {ext }}(x(u)) d u
$$

where $\alpha$ and $\beta$ are weighting parameters for the internal energy representing the stretching and bending, $\mathbf{M}^{\prime}(u), \mathbf{M}^{\prime \prime}(u)$ denotes $\left(\frac{\partial x(u)}{\partial u}, \frac{\partial y(u)}{\partial u}\right)^{T},\left(\frac{\partial^{2} x(u)}{\partial u^{2}}, \frac{\partial^{2} y(u)}{\partial u^{2}}\right)^{T}$, respectively, and $\gamma$ is the weight for the external energy $E_{\text {ext }}$.

The second term defines an important property of the snake, the smoothness of the contour. As smoothness is therefore contained in the definition of the spline [1], the functional for minimization can be rewritten as

$$
E=\int_{0}^{k} E_{e x t}(\mathbf{M}(u)) d u
$$

This approach shows some advantages to the classical description of snakes described in [1], [3], [7].

\section{Evolution of the Snake}

We construct the snake spline based on a manual initialization of the control points $P$ and let them evolute over time in a given strength field, where we used the GVF field. To find the solution of equation [2, the parametrized variable $\mathbf{M}(u)$ must be treated as a function of time $\mathbf{M}(u(t), t)$. The evolution of the control points under time can be described as [3]:

$$
\begin{aligned}
\frac{d P(t)}{d t}= & B^{+} \frac{d M(t)}{d t}=B^{+} F_{e x t}=-B^{+} \nabla I(M)= \\
& -B^{+} \nabla I(B P(t))=B^{+} V(B P(t))
\end{aligned}
$$




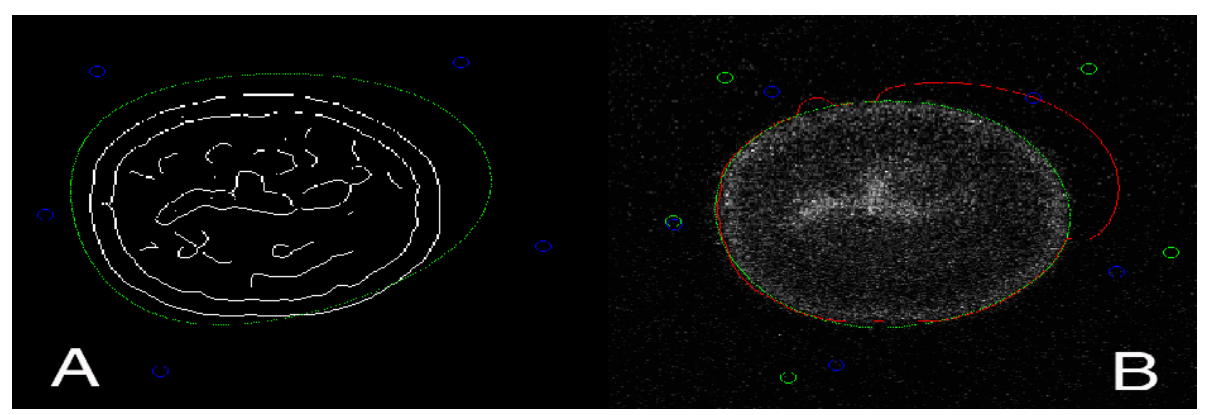

Fig. 2. Segmentation of nuclear membrane. (A) Initial snake spline defined by a set of five control points (blue). (B) The red curve representing the polygon snake and the green curve shows the snake spline respective with its control points (green)

$V(B P(t))$ are the contour points of the snake under the influence of the GVF vector field $V$. This ordinary differential equation can be solved by Runge and Kutta [5].

\section{Acknowledgements}

The authors want to thank D. Gerlich from the DKFZ Heidelberg and J. Ellenberg, EMBL Heidelberg for providing the nuclear envelope image shown in Fig. 2 We also thank B. Jähne for generously supporting this work.

\section{References}

1. Patrick Brigger, Jeff Hoeg, Michael Unser, B-Spline Snake: A Flexible Tool for Parametric Contour Detection, IEEE transaction on image processing, vol. 9, no. 9, 1484-1496, 2000

2. M. Kass, A. Witkin and D. Terzopoulos, Snakes: Active contour models, Int. J. Comput. Vis., pp. 321-331, 1987

3. Leitner F., Marque I., Lavallee P. and Cinquin P.: Dynamic segmentation: Finding edge with snake splines, Curves and Surfaces, pp. 279-284, Chamonix, France, 1990, 1991

4. Leitner F., Paillasson S., Ronot X., Demongeot J.: Dynamic functional and structural analysis of living cells: new tools for vital staining of nuclear DNA and for characterisation of cell motion, Acta Biotheor., 43(4), pp. 299-317, Dec. 1995

5. Press, Teukolsky, Vetterling, Flannery: Numerical Recipes in C, Second Edition, Cambridge University Press, 1999

6. Tvaruskó W., Bentele M., Misteli T., Rudolf R., Kaether C., Spector D.L., Gerdes H.H., Eils R.: Time-resolved analysis and visualization of dynamic processes in living cells, Proc. Natl. Acad. Sci. USA, Vol. 96, pp. 7950-7955, July 1999

7. Xenyang Xu and Jerry L. Prince, Snakes, Shapes and Gradient Vector Flow, IEEE Transaction on Image Processing, pp. 66-71, 1997 